\title{
Ureteral endometriosis: a rare and underdiagnosed cause of renal dysfunction
}

\author{
Swapna Yaramareddy ${ }^{1 *}$, Kundavi Shankar ${ }^{1}$, Abraham Kurien ${ }^{2}$, Thankam Varma ${ }^{1}$
}

\begin{abstract}
${ }^{1}$ Department of Obstetrics and Gynecology, Institute of Reproductive Medicine and Women's Health, Madras Medical Mission, Chennai, Tamil Nadu, India

${ }^{2}$ Department of Urology, Institute of Kidney Diseases, Urology and Organ Transplant, MMM Hospital, Chennai, Tamil Nadu, India
\end{abstract}

Received: 14 January 2018

Accepted: 10 February 2018

\section{*Correspondence:}

Dr. Swapna Yaramareddy,

E-mail: drswapnasrinath@gmail.com

Copyright: (C) the author(s), publisher and licensee Medip Academy. This is an open-access article distributed under the terms of the Creative Commons Attribution Non-Commercial License, which permits unrestricted non-commercial use, distribution, and reproduction in any medium, provided the original work is properly cited.

\begin{abstract}
Ureteral endometriosis is a serious localization of disease that can lead to urinary tract obstruction, hydroureteronephrosis and potential kidney loss. We describe three cases of ureteral endometriosis where there was documented renal function loss with subsequent workup and surgical intervention. Clinical suspicion and preoperative assessment may help with diagnosis and allows for a multidisciplinary pre-consultation. Surgical technique to treatment varies, but the goal is to salvage renal function and decrease disease burden. Laparoscopic surgical approach can be carried out successfully in the hands of an experienced laparoscopic surgeon. These cases illustrate varying surgical approaches tailored to management of ureteral endometriosis. Women in reproductive age presenting with pelvic pain diagnosed to have hydronephrosis must be adequately evaluated for ureteral endometriosis.
\end{abstract}

Keywords: Endometriosis, Laparoscopic surgery, Ureteroneocystotomy, Ureteral endometriosis, Ureterolysis

\section{INTRODUCTION}

Endometriosis is an estrogen-dependent chronic inflammatory condition characterized by the presence of endometrial like tissue outside the uterine cavity affecting $6 \%$ to $10 \%$ of women in reproductive age between $30-35$ years. ${ }^{1}$ Severe disease results in extensive adhesions and distortion of anatomy that leads to pain and infertility.

Urinary tract endometriosis is described as a rare entity with an incidence of $1-5.5 \%$ in patients with endometriosis. ${ }^{2,3}$ The incidence of ureteric endometriosis is increased in women with rectovaginal endometriotic nodules of more than $30 \mathrm{~mm}$ in size. ${ }^{4,5}$ Ureteral involvement is often limited to one ureter, commonly the left, and can lead to urinary tract obstruction, hydroureteronephrosis, and loss of renal function. There are estimates that $30 \%$ women with ureteral endometriosis will have $25 \%$ to $50 \%$ loss of nephrons at time of diagnosis of ureteral endometriosis, and an unknown number will then have loss of the kidney. ${ }^{6}$

We report our experience with ureteral endometriosis and subsequent varying surgical interventions.

\section{CASE REPORT}

\section{Case 1}

A 40-year-old lady with two previous normal vaginal deliveries presented with dysmenorrhoea and abnormal uterine bleeding, worsening since last 8 months. Ultrasound of the abdomen and pelvis showed an anterior wall fibroid of $3 \times 2 \mathrm{~cm}$, left endometriotic cyst of $6 \times 6$ $\mathrm{cm}$ with moderate left hydroureteronephrosis. Abdominal CT scan revealed dilatation of left renal pelvicalyceal 
system and ureter. Ureteric dilatation was noted up to level of S1 vertebra and there were no ureteric calculi. Anterior wall fibroid measuring $3.2 \times 2.6 \mathrm{~cm}$ was noted in the uterus.

The left ovary was enlarged in size measuring $6.1 \times 6.0 \times$ $4.8 \mathrm{~cm}$, right ovary was normal. Her blood urea was 29 $\mathrm{mg} / \mathrm{dl}$ and serum creatinine was $0.77 \mathrm{mg} / \mathrm{dl}$. Ethylenedicysteine (EC) Renogram was done which showed only $9 \%$ of functioning left kidney. Her CA 125 was $144.70 \mathrm{U} / \mathrm{ml}$, and CEA was normal. She underwent laparoscopic hysterectomy and laparoscopic nephrectomy. Intraoperatively uterus was bulky, right tube and ovary were normal. Left tube and ovary were not seen completely due to dense adhesions. Adhesiolysis followed by cystectomy was done, later proceeded to nephrectomy and hysterectomy. Uterus, bilateral ovaries and left kidney were delivered out through vaginal vault opening. Postoperative period was uneventful. She received three doses of inj. Leuprolide. Histopathological examination showed chronic papillary endocervicitis with non-secretory endometrium with uterine adenomyosis and leiomyoma. Left ovary and tube showed endometriosis. Left nephrectomy specimen showed chronic pyelonephritis with resection margin of ureter did not have significant lesion.

\section{Case 2}

35-year-old lady with primary subfertility underwent laparoscopic cystectomy at another center for symptomatic large endometriotic cyst measuring $7 \times 6 \mathrm{~cm}$ in the right ovary two years back. Two years later in view of persistent pelvic pain and dysmenorrhoea, she was revaluated. Her abdominal MRI revealed adenomyotic uterus with multiple endometriotic cysts bilaterally, largest cyst on the left side measuring $4.4 \times 5.3 \mathrm{~cm}$.

Right lower ureter showed tapering stenosis in pelvis at level of right ovary secondary to endometriotic stricture. She underwent right ureteric DJ stenting for right ureteric obstruction with hydroureteronephrosis. She visited our center for further evaluation and management. On evaluation, ultrasound abdomen showed persistent moderate right moderate HUN with stent in situ and multiple endometriotic cysts, largest left endometriotic cyst measuring $4 \times 5 \mathrm{~cm}$. EC renogram showed poorly functioning right kidney $(26 \%, 24 \mathrm{ml} / \mathrm{min})$ with obstructed drainage.

There was no functional obstruction on the left side. Her renal function test was normal, CA 125 was $51.6 \mathrm{U} / \mathrm{ml}$, CEA was normal, AMH was $1.64 \mathrm{ng} / \mathrm{ml}$. CT showed narrowing of lower ureter $2 \mathrm{~cm}$ above vesico ureteric junction for a length of $10 \mathrm{~mm}$ causing hydroureteronephrosis. She underwent cystoscopy and right laparoscopic Boari flap ureteroneocystotomy with DJ stent removal. She was started on Tablet Dienogest. She had a good follow up and her hydronephosis resolved.

\section{Case 3}

32-year-old lady, with history of having undergone a laparoscopic endometriotic cystectomy four years back for a symptomatic large endometriotic cyst measuring $9 \times 4 \times 5 \mathrm{~cm}$, presented to our center with severe dysmenorrhoea and primary subfertility.

On evaluation her AMH was $0.7 \mathrm{ng} / \mathrm{ml}, \mathrm{CA} 125$ was 295 $\mathrm{U} / \mathrm{ml}$, CEA was $1.56 \mathrm{ng} / \mathrm{ml}$. Her renal function tests were normal. MRI of abdomen and pelvis showed bulky adenomyotic uterus measuring $10 \times 8 \times 5.4 \mathrm{~cm}$ with bilateral endometriotic cysts (left $>$ right). Right cyst of 9.5 $\times 4.6 \mathrm{~cm}$ and left cyst of $16 \times 8.5 \mathrm{~cm}$. Gross hydroureteronephrosis involving the right kidney was seen. Minimal engulfment of rectum and recto-sigmoid junction was seen suggesting rectal infiltration. She underwent right DJ stenting for gross hydroureteronephrosis with laparoscopic bilateral ovarian endometriotic cystectomy. Intraoperatively, sigmoid colon adhesions to left ovary and uterus were noted. Pouch of Douglas was completely obliterated. Postoperatively, she was given two doses of Inj. Leuprolide depot $3.75 \mathrm{mg}$. She was treated with Tab Dienogest. She later underwent DJ stent removal after two months using a flexible cystoscope. On follow up her hydronephrosis has resolved.

\section{DISCUSSION}

Endometriosis is one of the most common gynaecologic disease in women, occurring between menarche and menopause. Endometriosis may present as a benign, albeit aggressive pathology with high local recurrences. It can involve the urinary tract and the ureter in particular. Urinary tract endometriosis is located in the bladder, ureter, kidney and urethra in $84 \%, 10 \%, 4 \%$, and $2 \%$ of cases, respectively. ${ }^{1,2}$ Involvement of the genitourinary tract has been reported to have the peak age of incidence being between 40 and 44 years of age. The disease need a high index of suspicion for both urologists and gynaecologists as the disease is insidious in onset with associated significant morbidity.

Although endometriosis was described as early as 1690 , its pathogenesis remains elusive. Multiple theories explaining the pathogenesis are the theory of retrograde menstruation, coelomic metaplasia theory, and the theory of lymphatic and venous spread. ${ }^{7}$ Where these theories have failed, stem cells have been linked to the pathogenesis of disease. ${ }^{8}$ None of the proposed theories independently elucidate the exact mechanism of development of endometriosis.

Endometriosis involves left pelvis more commonly than the right which can be explained by the anatomic differences in the pelvis. ${ }^{9}$ The distal segment of the ureters and bladder are the more frequently involved locations due to the proximity of the reproductive organs. Ureteral endometriosis is more likely to be associated 
with rectosigmoid lesions as opposed to bladder involvement.

Ureteral endometriosis is of two types, namely, extrinsic and intrinsic. In the extrinsic type, which is the most common, endometrial glandular and stromal tissue involve only the adventitia of the ureter or surrounding connective tissues, whereas the intrinsic type (20-30\% of cases) it involves the muscularis propria, lamina propria, or ureteral lumen. ${ }^{10}$ In the extrinsic form, patients have ureteric strictures, ureteral obstruction and hydronephrosis, because endometriosis lesions affect the ureter through adherence to the surrounding organs or structures.

Ureteral endometriosis is usually asymptomatic or presents with nonspecific symptoms. The presenting symptoms depend on whether the lesion is extrinsic or intrinsic. The common symptoms are severe dysmenorrhea, dyspareunia, and pelvic pain. Hematuria is usually noted in the intrinsic type. In most instances the disease is clinically silent. One-third of patients will have nonspecific symptoms consistent with pelvic endometriosis, and some patients will have symptoms of urgency, frequency, suprapubic and flank pain, hematuria, and dysuria. Specific genitourinary symptoms, as listed previously, in most instances, are related to endometriosis of the bladder and are seldom seen with ureteral involvement, thus making the diagnosis difficult.

Ureteral involvement can potentially lead to urinary tract obstruction with subsequent hydroureter and hydronephrosis. It has been reported that as many as $25 \%$ to $50 \%$ of nephrons are lost when there is evidence of ureteral endometriosis, at the time of diagnosis resulting in silent kidney loss. ${ }^{6}$ All our cases were consistent with extrinsic ureteral endometriosis with hydronephrosis. Although renal function test was normal, decreased functioning of the affected kidney was noted on EC renogram suggesting 'silent kidney loss' in these patients. A delay in diagnosis can lead to significant morbidity, such as a consequent worsening of hydronephrosis and further renal function loss. Therefore, early diagnosis is very important for this disease.

Multiple diagnostic tests can be used to confirm the existence of ureteral endometriosis. Ultrasonography, laparoscopy, intravenous urography, ureteroscopy with endoluminal ultrasound, CT scan and MRI are common diagnostic tools. Intravenous urography and CT scan are often used for their ability to localize laterality and level of ureteral constriction. Magnetic resonance imaging associated with magnetic resonance urography may be useful in detecting stricture of the pelvic ureter and hydroureteronephrosis. Solid nodules with speculated margins shows low-signal intensity on T2-weighted images that envelop the pelvic ureter, and also exploring all pelvic locations of endometriosis, thus it is the best imaging modality for the diagnosis of ureteral endometriosis. Magnetic resonance imaging has the ability to differentiate between intrinsic and extrinsic forms of ureteral endometriosis and has high specificity for bladder and renal lesions. ${ }^{1,12}$

Ureteroscopy is important in the diagnosis of intrinsic endometriosis. ${ }^{11}$ Laparoscopy and cystoscopy allow for direct visualization and obtaining endometrial tissues for pathological analysis.

The general principles of treatment for ureteral endometriosis should be considered to relieve the ureteral obstruction and symptoms, and to protect renal function. The therapeutic methods for endometriosis include medical and surgical therapy. Surgical therapy is the paramount consideration for the patients with hydronephrosis; a multidisciplinary team approach, including a skilled, advanced laparoscopic gynecologist, urologist, and colorectal surgeon play key roles in the successful treatment of extensive disease.

Laparoscopy is the gold standard for definitive diagnosis and surgical treatment of endometriosis. Laparoscopy offers many advantages over conventional laparotomy, namely a magnified view of the pelvis and greater exposure that allows for close examination and visualization of endometriotic implants. Larger implants or deep endometriotic nodules are best treated with resection. Careful destruction of all endometriotic implants is important to prevent recurrence.

Surgical interventions for relief of obstructive uropathy include ureterolysis, ureteroureterostomy, distal ureterectomy, and ureteral reimplantation or interposition of ileal segment between the ureter and bladder. Nephroureterectomy is a successful treatment alternative in refractory cases. ${ }^{13,14}$

Ureterolysis is often acceptable in cases of extrinsic, nonobstructive disease or for mild hydronephrosis. ${ }^{15}$ If there is any evidence of obstruction, then an excisional procedure should be done. If stenosis is limited to the ovarian fossa, distal ureter can be preserved, then ureteral resection and end-to-end anastomosis can be performed over a ureteral stent. Surgical approach is changed if the ureteral stenosis is close to the vesicoureteral junction and is extensive. The ureter is resected before the area of disease, and the proximal end is reimplanted into the bladder.

Our cases showcase three different surgical management options for treatment of ureteral endometriosis. In case 1, the decision was made in a multidisciplinary setting for a laparoscopic nephrouretectomy based on the patient's persistent symptoms and unlikelihood that the kidney could be salvaged. In case 2 , a right ureteral resection and ureteroneocystotomy was performed secondary to the distal location of diseased ureteral segment. In this case, it was thought that if the obstruction could be ameliorated, then renal function would be restored. In case 3, DJ stenting and bilateral endometriotic 
cystectomy with adhesiolysis was done for relieving obstructive hydroureteronephrosis. All three cases received post-operative medical management with GnRH agonists or progestins like dienogest to prevent postoperative recurrence. These cases illustrate that the approach to this rare form of the disease is multifaceted. Surgery is tailored to the specifics of localization of the disease and also the likelihood of recovery of renal function.

\section{CONCLUSION}

Endometriosis is a progressive disease. Ureteral endometriosis, though rare, is complicated by the potential to result in renal atrophy and functional loss. Thorough history, physical examination, and preoperative imaging can potentially help in the diagnosis. In young women with imaging findings of stricture of the pelvic ureter and hydroureteronephrosis, ureteral endometriosis should be considered. Multidisciplinary team approach is required for successful treatment of extensive disease. The surgical approach varies, but the goal is to salvage the renal system and decrease disease burden. In the hands of experienced, advanced laparoscopic surgeon, ureteral endometriosis can be treated effectively through minimally invasive techniques.

Funding: No funding sources Conflict of interest: None declared

Ethical approval: The study was approved by the Institutional Ethics Committee

\section{REFERENCES}

1. Giudice LC. Clinical practice. Endometriosis. N Engl J Med. 2010;362:238-98.

2. Comiter CV. Endometriosis of the urinary tract. Urol Clin North Am. 2002;29:625-35.

3. Abrao MS, Dias JA Jr, Bellelis P, Podgaec S, Bautzer CR, Gromatsky C. Endometriosis of the ureter and bladder are not associated diseases. Fertil Steril. 2009;91:1662-7.

4. Donnez J, Nisolle M, Squifflet J. Ureteral endometriosis: a complication of rectovaginalendometriotic (adenomyotic) nodules. Fertil Steril. 2002;77:32-7.

5. Kondo W, Branco AW, Trippia $\mathrm{CH}$, Ribeiro R, Zomer MT. Retrocervical deep infiltrating endometriotic lesions larger than thirty millimeters are associated with an increased rate of ureteral involvement. J Minim Invasive Gynecol. 2013;20:100-3.

6. Horn LC, Do Minh M, Stolzenburg JU. Intrinsic form of ureteral endometriosis causing ureteral obstruction and partial loss of kidney function. Urol Int. 2004;73:181-4.

7. Nap AW, Groothuis PG, Demir AY, Evers JL, Dunselman GA. Pathogenesis of endometriosis. Best Pract Res Clin Obstet Gynaecol. 2004;18:233-44.

8. Sasson IE, Taylor HS. Stem cells and the pathogenesis of endometriosis. Ann N Y Acad Sci. 2008;1127:106-15.

9. Chapron C, Chopin N, Borghese B, Foulot H, Dousset B, Vacher-Lavenu MC, et al. Deeply in ltrating endometriosis: pathogenetic implications of the anatomical distribution. Hum Reprod. 2006;21:1839-45.

10. Gabriel B, Nassif J, Trompoukis P, Barata S, Wattiez A. Prevalence and management of urinary tract endometriosis: a clinical case series. Urol. 2011;78:1269-74.

11. Kinkel K, Frei KA, Balleyguier C, Chapron C. Diagnosis of endometriosis with imaging: a review. Eur Radiol. 2006;16:285e98.

12. Balleyguier C, Roupret M, Nguyen T, Kinkel K, Helenon O, Chapron C. Ureteral endometriosis: the role of magnetic resonance imaging. J Am Assoc Gynecol Laparosc. 2004;11:530e6.

13. Nezhat C, Nezhat F, Nezhat CH, Nasserbakht F, Rosati M, Seidman DS. Urinary tract endometriosis treated by laparoscopy. Fertil Steril. 1996;66:920e4.

14. Yohannes P. Ureteral endometriosis. J Urol. 2003;170:20e5.

15. Ghezzi F, Cromi A, Bergamini V, Serati M, Sacco A, Mueller MD. Outcome of laparoscopic ureterolysis for ureteral endometriosis. Fertil Steril. 2006;86:418e22.

Cite this article as: Yaramareddy S, Shankar K, Kurien A, Varma T. Ureteral endometriosis: a rare and underdiagnosed cause of renal dysfunction. Int $\mathrm{J}$ Reprod Contracept Obstet Gynecol 2018;7:1281-4. 